DEUTSCHE DEMOKRATISCHE REPUBLIK

DEUTSCHE AKADEMIE

DER LANDWIRTSCHAFTSWISSENSCHAFTEN ZU BERLIN

\title{
ARCHIV \\ FÜR \\ GARTENBAU
}

XI. BAND - HEFT 7

1963

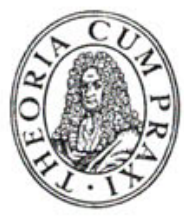

A K A D E M I E - V E R LA G B E R L I N 

DEUTSCHE DEMOKRATISCHE REPUBLIK

DEUTSCHE AKADEMIE

DER LANDWIRTSCHAFTSWISSENSCHAFTEN ZU BERLIN

\section{ARCHIV FÜR GARTENBAU}

XI. BAND - HEFT 7

1963

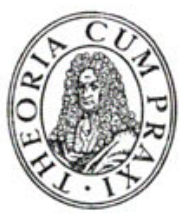

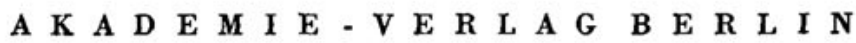

